\title{
Time-dependent gravitating solitons in five-dimensional warped space-times
}

\author{
Massimo Giovannini* \\ Centro "Enrico Fermi," Compendio del Viminale, Via Panisperna 89/A, 00184 Rome, Italy, \\ and Department of Physics, Theory Division, CERN, 1211 Geneva 23, Switzerland
}

(Received 5 October 2007; published 17 December 2007)

\begin{abstract}
Time-dependent soliton solutions are explicitly derived in a five-dimensional theory endowed with one (warped) extra dimension. Some of the obtained geometries, everywhere well defined and technically regular, smoothly interpolate between two five-dimensional anti-de Sitter space-times for a fixed value of the conformal time coordinate. Time-dependent solutions containing both topological and nontopological sectors are also obtained. Supplementary degrees of freedom can be also included and, in this case, the resulting multisoliton solutions may describe time-dependent kink-antikink systems.
\end{abstract}

DOI: 10.1103/PhysRevD.76.124017

PACS numbers: 04.50.+h, 05.45.Yv, 11.10.Kk, 11.25.Mj

\section{PHYSICAL MOTIVATIONS}

Time-dependent soliton solutions arise typically in $(1+$ 1) space-time dimensions by appropriately boosting static solutions. This observation is due to the pioneering work of various authors [1-3] (see also [4,5] and references therein for an extended introduction) whose main focus was on the sine-Gordon system. The static solutions of the $(1+1)$ dimensional system only involved one scalar degree of freedom. Furthermore, the geometry was always static and Minkowskian both in the case of static and timedependent solutions. The boosted solutions could be simply interpreted, from the physical point of view, as traveling solitons which could even illustrate, with appropriate boundary conditions (see, for instance, [3]), the elastic collision of two profiles.

In $(1+1)$ dimensions, static multisoliton solutions are also known to exist. The obtainable solutions can be categorized into two basic groups. To the first set belong solutions containing two (or more) topological defects. The second group of solutions contemplates two (or more) defects of which at least one is a nontopological soliton. In the figurative language of some of the authors discussing these profiles they have been dubbed as baglike solutions [6-8]. According to the same language it is also possible to achieve trapping-bag configurations where a topological kink is trapped by a nontopological bag (see also $[9,10]$ where trapping-bag solutions appear in the framework of the so-called MIT-bag and SLAC-bag models of extended hadrons). The interest of multisoliton solutions in $(1+1)$ dimensions persists even in recent years and various interesting integration methods have been tailored to deal, specifically, with nonlinear multifield equations [11-13].

The main theme of the present paper will not be $(1+1)$ dimensional systems but rather five-dimensional gravitat-

*massimo.giovannini@cern.ch ing soliton solutions. It is nonetheless inspiring to bear in mind the aforementioned observations since the topic of the present paper will be time-dependent generalizations of static soliton solutions in five-dimensional warped spacetimes.

Gravitating solitons in higher dimensions can be used to model, for instance, five-dimensional domain walls where the extra (bulk) coordinate is infinite and where the spacetime geometry is consistently included as a solution of the appropriate higher-dimensional gravitational field equations. Single gravitating kinks in five dimensions [1420] have been discussed (see also [21,22]). Following the analogy suggested at the beginning of the present script, it is possible to find baglike solutions in five-dimensional geometries with well-defined $\mathrm{AdS}_{5}$ limit for large values of the bulk radius. It has been shown that multisoliton solutions arise naturally also in a five-dimensional context and fully analytic solutions have been reported, always in warped geometries, where the field profiles describe both kink-antikink systems and trapping-bag solutions [23].

These discussions have been conducted, initially, in the framework of five-dimensional Einstein-Hilbert gravity. Possible extensions of this approach include

(i) the modification of the underlying gravitational theory (for instance from Einstein-Hilbert gravity to Gauss-Bonnet or Brans-Dicke gravity);

(ii) the increase of the dimensionality of the space-time (for instance from kinks in five dimensions to Abelian vortices in six dimensions);

(iii) the increase in the number of defects (for instance from single-defect solutions to multidefect solutions).

One of the distinctive aspects of the aforementioned attempts is that the obtained field profiles are thick, i.e. they have an internal structure and they are obtained as a consistent solution of the underlying gravitational theory. In this sense the nature of the physical system greatly differs 
from their $(1+1)$-dimensional counterpart. Particularly interesting situations are the ones where the geometry is completely regular ${ }^{1}$ and it has well-defined $\mathrm{AdS}_{5}$ limits for large absolute value of the bulk radius.

The gravity theory employed in the actual constructions plays a relevant role in the physical properties of the solutions. As already mentioned, the obvious choice is to embed the defects in a higher-dimensional Einstein-Hilbert gravity theory (see, for instance, [20] and references therein). However, also different choices are equally plausible. For instance one might choose to select a theory where the Einstein-Hilbert terms are nonminimally coupled to the scalar degree of freedom [24-27]. Gravitating kinks have been also studied in quadratic gravity theories where the Einstein-Hilbert action is complemented by the Gauss-Bonnet combination [28-31]. It is finally possible to combine these two options and discuss the possibility of higher-dimensional solutions where the Einstein-Hilbert action is complemented by the GaussBonnet combination and the dilaton couples explicitly to the curvature in the string frame metric. This possibility has been developed in [32-34].

Another arena for thick defects involves also the increase of the dimensionality of the space-time. In the framework of the Abelian-Higgs model it is possible to find thick string solutions in a six-dimensional space-time with well-defined $\mathrm{AdS}_{6}$ limit far from the vortex and a Minkowskian limit close to the core of the defect. These solutions may arise, with different features, both within Einstein-Hilbert gravity [35-39] and within Gauss-Bonnet gravity [40]. Another example is represented by higherdimensional hedgehogs which are present in the case of seven (warped) dimensions (see [41,42] and references therein). Finally, there exist, always in a five-dimensional context, solutions where the solitons have nontopological properties [43], i.e. the static fields have a baglike profile as a function of the bulk radius.

In the present paper the main theme will be to investigate time-dependent soliton solutions that are inspired by the symmetries of the static solutions. There is the hope, in this context, of finding new classes of cosmological models that do not follow in the class of separable solutions that have been already exploited in the five-dimensional case. The latter solutions are typically obtained in the presence of thin brane configurations that arise as Dirac delta functions in the energy-momentum tensor of the brane. Typically these solutions are singular at the location of the brane and the defects, being thin, do not have internal structure. In different words we can say the interest of the present exercises resides mainly in the fact that time-dependent solitonic solutions can be eventually used to describe, for a

\footnotetext{
${ }^{1}$ In this paper, when talking about regular geometry, we will always refer to the case when the relevant curvature invariants are finite for all values of the coordinates lying in the domain of definition of the solutions.
}

fixed value of the bulk radius, higher-dimensional cosmological models which are, really and truly, stemming from thick gravitating defects or, for short, thick branes.

To introduce the discussion in more specific terms consider the static solutions of the following five-dimensional theory ${ }^{2}$ :

$$
S=\int d^{5} x \sqrt{|G|}\left[-\frac{R}{2 \kappa}+\frac{1}{2} G^{A B} \partial_{A} \varphi \partial_{B} \varphi-V(\varphi)\right]
$$

in the case of the line element

$$
d s^{2}=G_{A B} d x^{A} d x^{B}=a^{2}(w)\left[\eta_{\mu \nu} d x^{\mu} d x^{\nu}-d w^{2}\right],
$$

where $w$ is the bulk coordinate, $a(w)$ is the (static) warp factor, and $\eta_{\mu \nu}$ is the four-dimensional Minkowski metric. ${ }^{3}$ Consider then, a specific solution of this theory (see $[14,16,18]$ and references therein), i.e.

$$
a(w)=\frac{1}{\sqrt{\lambda^{2} w^{2}+1}}, \quad \varphi=\sqrt{\frac{3}{\kappa}} \arctan (b w),
$$

arising when the potential $V(\phi)$ takes the following trigonometric form:

$$
V(\varphi)=\frac{3 \lambda^{2}}{2 \kappa}\left[1-5 \sin ^{2} \Phi\right], \quad \Phi=\sqrt{\frac{3}{\kappa}} \varphi
$$

From Eqs. (1.3) and (1.4) we can infer two kinds of solutions:

(i) boosted solutions that can be obtained by replacing $w$ with $\gamma(u)(w+u t)$ or with $\gamma(u)(w-u t)$ (where $\left.\gamma(u)=\left(1-u^{2}\right)^{-1 / 2}\right)$

(ii) time-shifted solutions.

The first class of solutions describes kinks that travel along the fifth coordinate and they will be discussed a bit later in this paper. The second class of solutions can be more directly interpreted as a time-dependent cosmological model for a fixed value of the bulk coordinate (that can be chosen to be, for instance, at $w=0$ ). The trouble with this second class of solutions is that, in general, they will not be diagonal. Indeed, from Eqs. (1.2), (1.3), and (1.4) it is possible to deduce that a time-dependent solution of the theory can be found, always for the potential (1.4), for a

\footnotetext{
${ }^{2}$ In Eq. (1.1) $\kappa=8 \pi G_{5}=8 \pi / M^{3}$ and $G_{5}$ is the fivedimensional Newton constant.

${ }^{3}$ Conventions: capital Latin indices run over the whole dimensionality of the space-time while Greek indices run over the fourdimensional space-time; Latin (lowercase) indices run over the three-dimensional spatial geometry. The signature of the metric is mostly minus, i.e. $(+,-,-,-,-)$.
} 
line element of the type ${ }^{4}$

$$
d s^{2}=a^{2}(w, \tau)\left[d \tau^{2}-d \vec{x}^{2}-d w^{2}-2 d w d \tau\right]
$$

where

$$
\begin{aligned}
a(w, t) & =\frac{1}{\sqrt{\lambda^{2}(w+\tau)^{2}+1}}, \\
\varphi & =\sqrt{\frac{3}{\kappa} \arctan [\lambda(w+\tau)] .}
\end{aligned}
$$

The easiest way of getting convinced of this statement is by using the symmetry of the metric and then, as a crosscheck, to verify that the transformed metric is a solution of the action (1.1) with the potential given in Eq. (1.4). Indeed, the Ricci tensor obtained from Eqs. (1.5) and (1.6) has covariant components that can be computed to be

$$
\begin{aligned}
R_{00} & =2 \lambda^{2} \frac{1+2 \lambda^{2}(w+\tau)^{2}}{\left[1+\lambda^{2}(w+\tau)^{2}\right]^{2}}, \\
R_{i j} & =\lambda^{2} \frac{1-4 \lambda^{2}(w+\tau)^{2}}{\left[1+\lambda^{2}(w+\tau)^{2}\right]^{2}} \delta_{i j}, \\
R_{w w} & =4 \lambda^{2} \frac{1-\lambda^{2}(w+\tau)^{2}}{\left[1+\lambda^{2}(w+\tau)^{2}\right]^{2}}, \\
R_{w 0} & =R_{0 w}=4 \lambda^{2} \frac{1-\lambda^{2}(w+\tau)^{2}}{\left[1+\lambda^{2}(w+\tau)^{2}\right]^{2}} .
\end{aligned}
$$

By contraction of the Einstein equations derived from Eq. (1.1), the relevant system can be recast in the following form:

$$
\begin{gathered}
R_{A B}=\kappa\left[\partial_{A} \varphi \partial_{B} \varphi-\frac{2}{3} V\right], \\
G^{A B} \nabla_{A} \nabla_{B} \varphi+\frac{\partial V}{\partial \varphi}=0,
\end{gathered}
$$

where $\nabla_{A}$ is the covariant derivative computed in terms of the five-dimensional metric. Using the field profile appearing in Eq. (1.6) and the potential of Eq. (1.4) into Eqs. (1.8)

\footnotetext{
${ }^{4}$ In what follows the time coordinate shall be denoted by $\tau$. This is motivated by the fact, as it will be shown, that the geometries written in the parametrization adopted in the present script reduce to what we would call a conformally flat metric for a fixed value of the bulk coordinate. In this case the time coordinate, i.e. $\tau$ will be what is customarily called, in cosmological applications, the conformal time coordinate (as opposed to the cosmic time coordinate where all the clocks are synchronized). This is the reason why we prefer to use $\tau$ rather than $t$ to denote the time.
}

and (1.9) the system is satisfied and the solution is proven to exist. Notice that the off-diagonal form of the metric reported in Eq. (1.5) is essential to match the off-diagonal components of the Ricci tensor (i.e. $R_{0 w}$ and $R_{w 0}$ ) which are now nonvanishing since the warp factor depends explicitly upon time. Indeed, from Eq. (1.5), the $0 w$ and $w 0$ components of Eq. (1.8) lead also to a generalized momentum constraint that mixes derivatives of the field profile with respect to $w$ and with respect to $\tau$. The momentum constraint is solved iff the metric is in the form of Eq. (1.5), i.e. off-diagonal.

This example is, at the same time, tangential and inspiring. It is tangential since, as already remarked, the obtained geometry is necessarily off-diagonal and, therefore, difficult to interpret as a cosmological metric at fixed value of the bulk coordinate. It is, however, also inspiring since it suggests that it might not be hopeless to find timedependent soliton solutions leading to a nonseparable form of the warp factor. By separable form of the warp factor we mean solutions where $a(w, \tau)$ can be written as the product of two functions one depending solely upon $w$ and the other depending solely upon $\tau$. Separable solutions are normally discussed in the framework of thin brane models but, as we will argue, they are rather unnatural in the case of thick defects.

The plan of the forthcoming discussion will therefore be the following. In Sec. II we will look for diagonal (timedependent and nonseparable) solutions in the fivedimensional case and with a single scalar profile. In Sec. III this discussion will be generalized to the case of multidefects. Section IV contains some complementary material on traveling solitons. Finally Sec. V contains our concluding remarks.

\section{TIME-DEPENDENT GRAVITATING DEFECTS}

To improve on the off-diagonal ansatz shown to satisfy the whole system of equations supported by timedependent (gravitating) profiles discussed in the introduction, it is appropriate to consider the following ansatz:

$$
\begin{aligned}
d s^{2} & =G_{A B} d x^{A} d x^{B} \\
& =a^{2}(w, \tau)\left[d \tau^{2}-d \vec{x}^{2}\right]-b^{2}(w, \tau) d w^{2} .
\end{aligned}
$$

Using Eq. (2.1) for the calculation of the Ricci tensor and of the Christoffel connections the various components of Eq. (1.8) easily become, after some algebra:

$$
\begin{aligned}
\frac{a^{2}}{b^{2}} & {\left[\mathcal{H}^{\prime}+4 \mathcal{H}^{2}-\mathcal{H} \mathcal{F}\right]-[\dot{F}+3 \dot{H}+F(F-H)] } \\
& =\kappa\left[\dot{\varphi}^{2}-\frac{2}{3} V a^{2}\right]
\end{aligned}
$$




$$
\begin{gathered}
-\frac{a^{2}}{b^{2}}\left[\mathcal{H}^{\prime}+4 \mathcal{H}^{2}-\mathcal{H} \mathcal{F}\right]+\left[\dot{H}+2 H^{2}+H F\right] \\
=\frac{2}{3} \kappa V a^{2}, \\
\frac{b^{2}}{a^{2}}\left[\dot{F}+F^{2}+2 H F\right]-4 \mathcal{H}^{\prime}+4 \mathcal{H}(\mathcal{F}-\mathcal{H}) \\
=\kappa\left[\varphi^{\prime 2}+\frac{2}{3} V b^{2}\right], \\
\mathcal{H}-4 H^{\prime}+3 F \mathcal{H}=\kappa \dot{\varphi} \varphi^{\prime} .
\end{gathered}
$$

The notations employed in Eqs. (2.2), (2.3), (2.4), and (2.5) are the following:

(i) the prime denotes a derivation with respect to the bulk radius $w$;

(ii) the overdot denotes a derivation with respect to the conformal time coordinate $\tau$;

(iii) $\mathcal{H}$ and $\mathcal{F}$ are related with the derivatives of $a$ and $b$ with respect to $w$, i.e. more specifically,

$$
\mathcal{H}(w, \tau)=\frac{\partial \ln a}{\partial w}, \quad \mathcal{F}(w, \tau)=\frac{\partial \ln b}{\partial w} ;
$$

(iv) $H$ and $F$ are related with the derivatives of $a$ and $b$ with respect to $\tau$, i.e. more specifically,

$$
H(w, \tau)=\frac{\partial \ln a}{\partial \tau}, \quad F(w, \tau)=\frac{\partial \ln b}{\partial \tau} .
$$

Equations (2.2), (2.3), and (2.4) follow, respectively, from the $(00),(i=j)$, and $(w w)$ components of Eq. (1.8) while Eq. (2.5) follows from the $(0 w)$ component of Eq. (1.8). Finally, Eq. (1.9) leads, within the notations expressed by Eqs. (2.6) and (2.7), to the following explicit relation

$$
\begin{array}{r}
\ddot{\varphi}-\frac{a^{2}}{b^{2}} \varphi^{\prime \prime}+(2 H+F) \dot{\varphi}-\frac{a^{2}}{b^{2}}(4 \mathcal{H}-\mathcal{F}) \varphi^{\prime} \\
+\frac{\partial V}{\partial \varphi} a^{2}=0 .
\end{array}
$$

If the dependence upon $\tau$ and $w$ would be separable (i.e. $a(w, \tau)=\alpha(\tau) \tilde{a}(w)$ and $b(w, \tau)=\beta(\tau) \tilde{b}(w)$ ), then, necessarily, $\mathcal{H}$ and $\mathcal{F}$ will only be functions of the bulk radius (i.e. $\mathcal{H}(w)$ and $\mathcal{F}(w)$ ), while $H$ and $F$ will only be functions of the conformal time coordinate (i.e. $H(\tau)$ and $F(\tau)$ ). The constraint of Eq. (2.5) can then be trivially satisfied by requiring either $F=0$ (i.e. $\beta$ constant in $\tau$ ) or $\mathcal{H}=0$ (i.e. $\tilde{a}$ constant in $w$ ). This situation is, however, not generic.

It is appropriate to mention that by taking linear combinations of some of the above equations, the resulting expressions can be put in a more friendly form. In particular, by summing up Eqs. (2.2) and (2.3) we do get

$$
\dot{\varphi}^{2}=\frac{1}{\kappa}\left[2 H^{2}+2 H F-F^{2}-\dot{F}-2 \dot{H}\right] .
$$

By combining Eqs. (2.3) and (2.4) we do get instead

$$
\begin{aligned}
\varphi^{\prime 2}= & \frac{3}{\kappa}\left(\mathcal{H} \mathcal{F}-\mathcal{H}^{\prime}\right) \\
& +\frac{1}{\kappa} \frac{a^{2}}{b^{2}}[(\dot{F}-\dot{H})+(F+2 H)(F-H)] .
\end{aligned}
$$

Consider then the following form of the warp factors:

$$
\begin{aligned}
& a(w, \tau)=\frac{1}{\sqrt{\lambda^{2}(w+\tau)^{2}+1}}, \\
& b(w, \tau)=\frac{\epsilon}{\sqrt{\lambda^{2}(w+\tau)^{2}+1}},
\end{aligned}
$$

where $\epsilon$ is both constant in $w$ and in $\tau$. It can be verified that Eq. (2.11) satisfies the whole system of equations provided the scalar field and the potential are

$$
\begin{gathered}
\varphi(w, \tau)=\sqrt{\frac{3}{\kappa}} \arctan [\lambda(w+\tau)], \\
V(\varphi)=\frac{3 \lambda^{2}}{2 \kappa} \frac{\epsilon^{2}-1}{\epsilon^{2}}\left(5 \sin ^{2} \Phi-1\right), \quad \Phi=\sqrt{\frac{\kappa}{3}} \varphi .
\end{gathered}
$$

If $\epsilon \rightarrow 1$ the solution does not reduce to a static limit but rather it reduces to a free solution with vanishing potential (i.e. $V(\varphi)=0$ ) where the explicit expression of $\varphi$ is still given by Eq. (2.12). Again, the solution $\epsilon=1$ does not have a static analog since the time dependence is essential. In other words $\Phi(w)=\arctan \lambda w$ is not a solution of the system if we set the time dependence to zero and $V(\Phi)=0$ from the very beginning. The solution of Eqs. (2.11), (2.12), and (2.13) can be generalized to a family of solutions characterized by an integer parameter $\mu$. The solution can be written as

$$
\begin{aligned}
a(w, \tau) & =\frac{1}{\left[\lambda^{2 \mu}(w+\tau)^{2 \mu}+1\right]^{(1 / 2 \mu)}}, \\
b(w, \tau) & =\epsilon a(w, \tau),
\end{aligned}
$$

where, as before, $\epsilon$ is a constant. The form of the warp factor given in Eq. (2.14) solves the whole system provided

$$
\begin{aligned}
\varphi(w, \tau)= & \sqrt{\frac{3(2 \mu-1)}{\kappa}} \arctan \left[\lambda^{\mu}(w+\tau)^{\mu}\right], \\
V(\varphi)= & \frac{3 \lambda^{2}}{2 \kappa}\left(\frac{\epsilon^{2}-1}{\epsilon^{2}}\right) \Lambda^{(\mu-1 / \mu)}(\varphi) \\
& \times[(2 \mu+3) \Lambda(\varphi)-(2 \mu-1)],
\end{aligned}
$$

where $\mu \geq 1$ and 


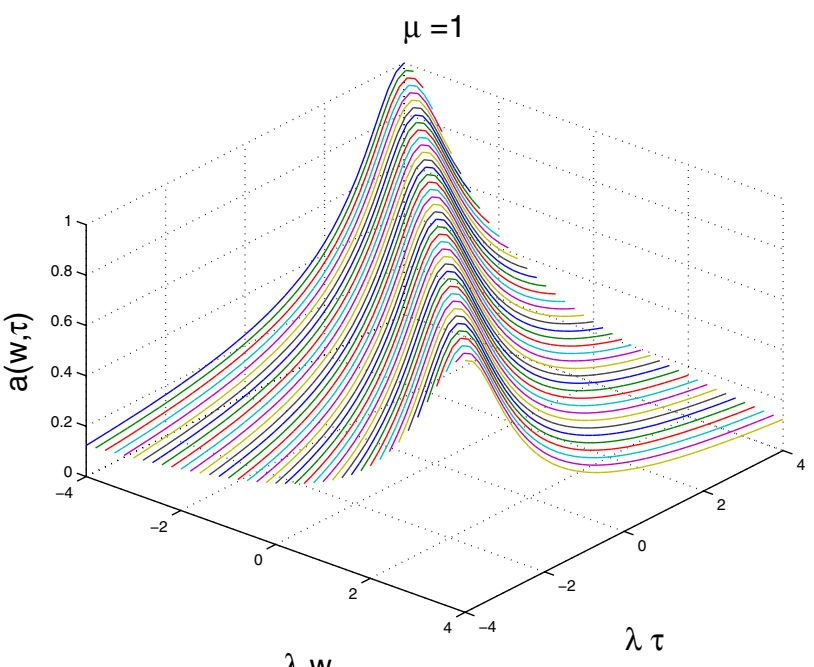

$\lambda w$

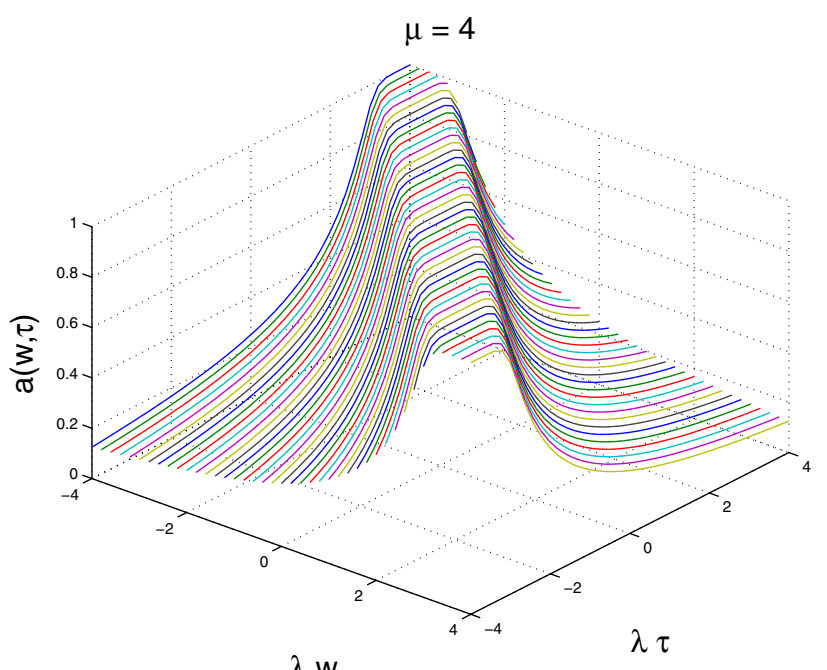

$\lambda w$

FIG. 1 (color online). The warp factors for different values of the integer $\mu$.

$$
\Lambda(\varphi)=\sin ^{2} \Phi, \quad \Phi=\sqrt{\frac{\kappa}{3(2 \mu-1)} \varphi . ~}
$$

In the case $\mu=1$ the solution expressed by Eqs. (2.14), (2.15), and (2.16) goes exactly to the solution of Eqs. (2.11), (2.12), and (2.13). Again, in the limit $\epsilon=1$, Eqs. (2.14) and (2.15) still solve the system with vanishing potential.

Different values of $\mu$ imply different properties for the topology of the field solution. This point is illustrated in Figs. 1-3 where the warp factors and the field profiles are reported for different values of the integer $\mu$ that appears in Eqs. (2.14), (2.15), and (2.16). According to Fig. 1, when $\mu$ increases from 1 to 4 the warp factor becomes broader in the $(w, t)$ plane (recall that $b(w, \tau)=\epsilon a(w, \tau))$. For a fixed value of the conformal time coordinate, say $\tau=0$, $a(w, 0) \rightarrow|\lambda w|^{-1}$ for $|\lambda w| \rightarrow \infty$ and $b(w, 0) \rightarrow \epsilon|\lambda w|^{-1}$.

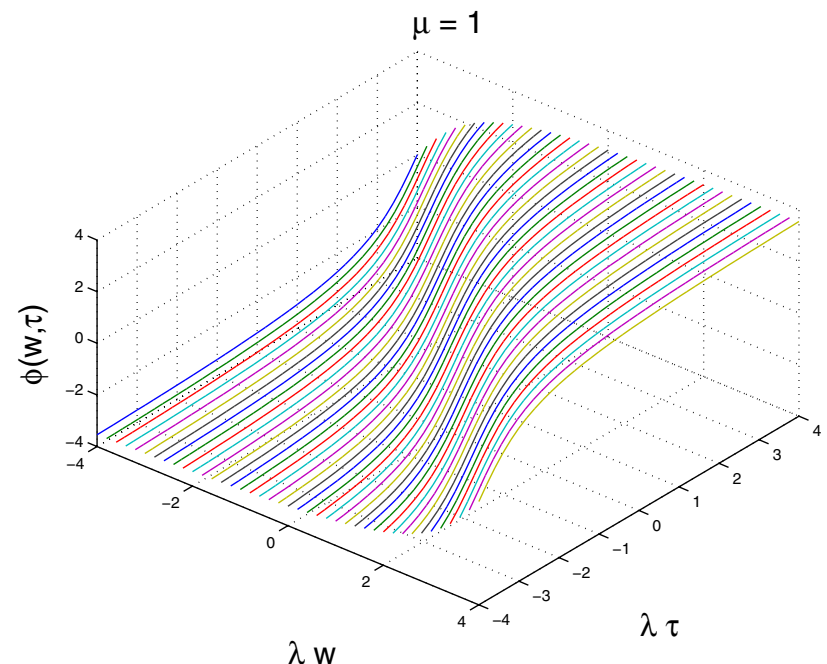

In this case the space-time geometry can be viewed, for large absolute value of the bulk radius, as a slight deformation of $\mathrm{AdS}_{5}$. The deformation parameter is exactly $\epsilon$, which is dimensionless. As soon as $\epsilon \rightarrow 1$ the exact $\mathrm{AdS}_{5}$ limit is recovered for $|w| \rightarrow \infty$. The geometry is always regular and all the curvature invariants do not have any pole for any finite values of either $w$ or $\tau$. In the limit $\epsilon \rightarrow 1$ the Weyl invariant vanishes. A change in the value of $\mu$ does affect crucially the topological nature of the field profile. This aspect can be simply understood from Eq. (2.15). If $\mu$ is even the field profile interpolates between two different minima of the underlying potential and it has the characteristic kinklike shape. In Fig. 2 this aspect is illustrated for two different odd values of $\mu$, i.e. $\mu=1$ and $\mu=3$. Notice a slight difference between the case $\mu=1$ and the case $\mu=3$. In the case $\mu=3$ (see Fig. 2, plot at the right) the profile flattens around the origin of the

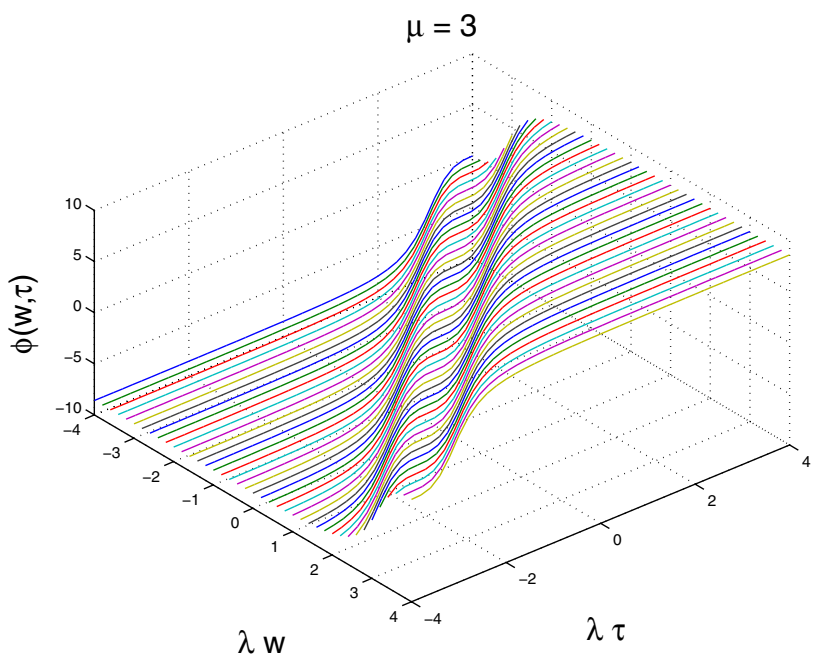

FIG. 2 (color online). The field profile for odd values of $\mu$. Natural gravitational units $2 \kappa=1$ have been adopted. 

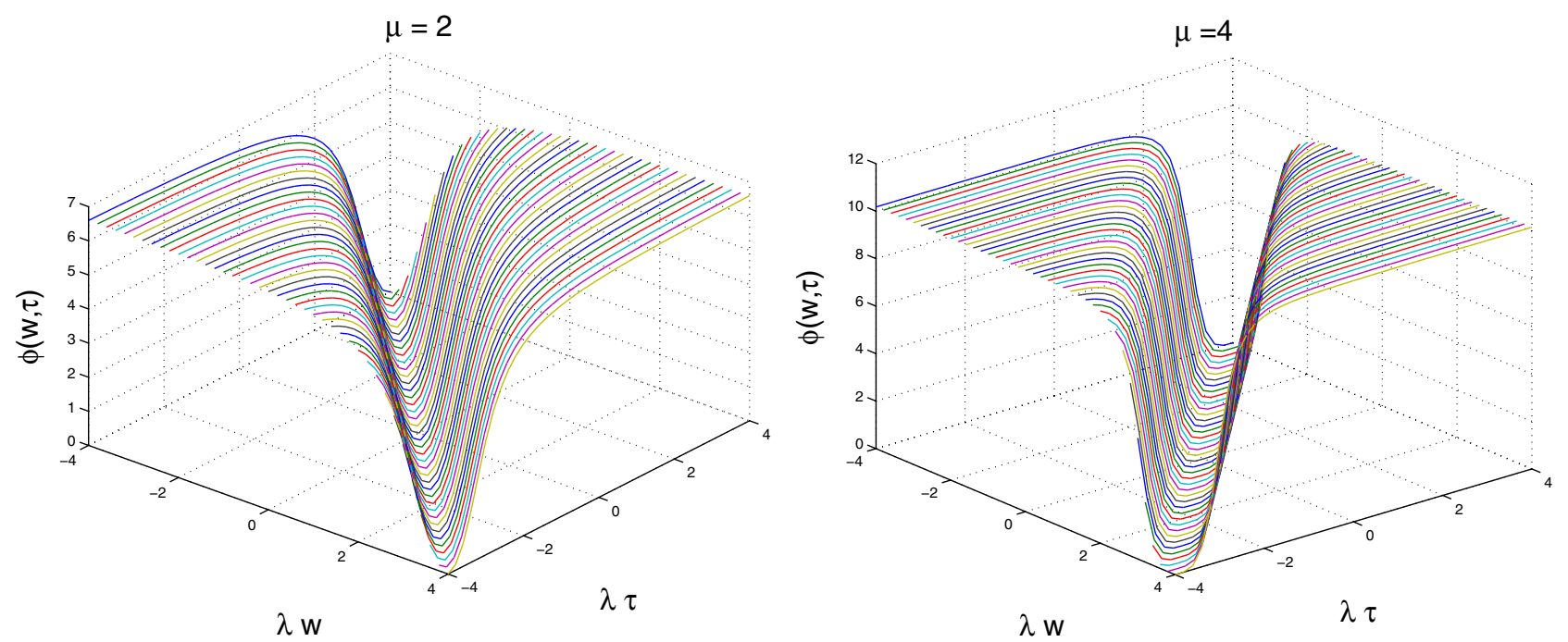

FIG. 3 (color online). The field profiles for even values of $\mu$ are reported in the natural gravitational units introduced in Fig. 2 .

coordinate system. In fact, in the case $\mu=1$ the first derivative of $\Phi(w, \tau)$ (both with respect to $w$ and $\tau$ ) is not vanishing at the origin, i.e. for $w=\tau=0$. On the contrary, at the same point, in the case $\mu=3$ the first and second derivatives of $\Phi(w, \tau)$ are both equal to 0 and the only nonvanishing derivative at the origin will be the third one. Besides this point we can safely say that, when $\mu$ is odd, the field profile exhibits a topological nature. Conversely, when $\mu$ is even, the field profile does not have a kinklike structure but rather a baglike structure signaling that the corresponding field profile is nontopological. This aspect is usefully illustrated in Fig. 3 where $\varphi(w, \tau)$ is reported in the cases $\mu=2$ and $\mu=4$. As it appears from Eq. (2.15), when $\mu$ is even, the field profile tends to the same constant values when $w$ and $\tau$ are large in absolute values. As far as the cosmological properties of the solutions are concerned, it is useful to look at Fig. 4

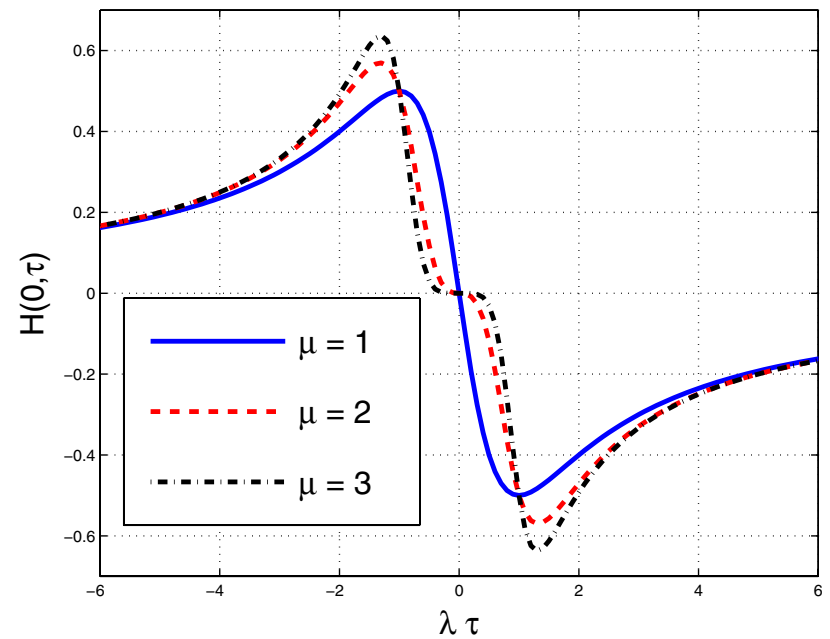

where $H$ and $\dot{H}$ are reported as a function of the conformal time coordinate $\tau$. It is clear from Fig. 4 that, for the fixed value of the bulk radius, say $w=0, H(\tau)$ changes sign once and $\dot{H}$ changes sign twice. This behavior is typical of a well-known class of cosmological scenarios called bounces. Bouncing solutions in the Einstein frame (which is the one adopted in the present analysis) are solutions where the Hubble rate flips its sign [44,45] (see also [46] and references therein). The bouncing behavior obtained in the present solutions and illustrated in Fig. 4 is, at the same time, unrealistic and encouraging. It is unrealistic since a physical bounce should have features that are opposite to the ones exhibited by Fig. 4. In other words a realistic bounce should have $H<0$ for $\tau<0$ and $H>0$ for $\tau>0$. This type of evolution would imply that the contraction took place in the far past (for $\tau<0$ ) while today, as we know, the Universe expands rather than contracting. In

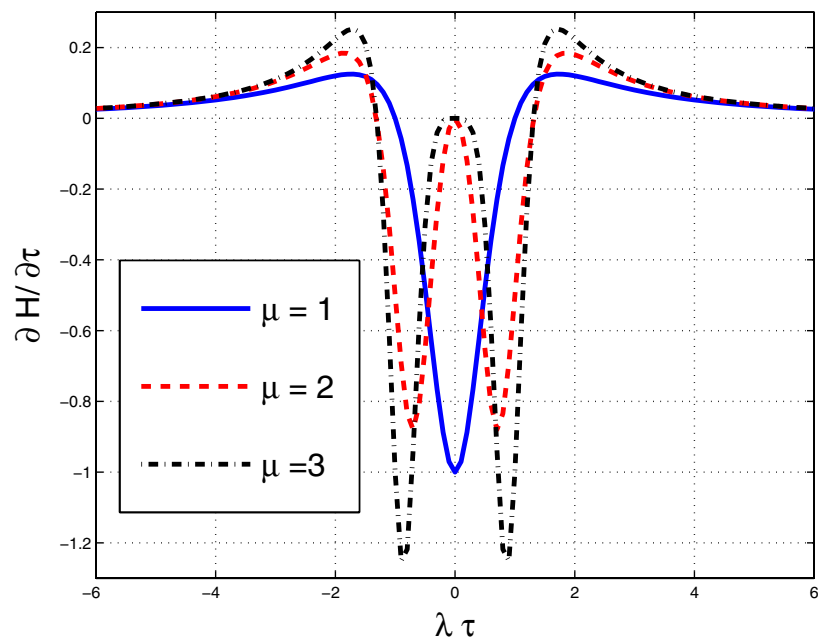

FIG. 4 (color online). The conformal time evolution of $H$ and $\dot{H}$ is illustrated at a fixed value of the bulk radius and for different values of $\mu$. 
Fig. 4 the behavior of $H$ (and $\dot{H}$ ) is opposite: the Universe expands in the past and contracts in the future, which is unrealistic. At the same time, however, the obtained results are encouraging since we showed, analytically, that a bouncing behavior arises naturally.

It is relevant to mention that the only separable solutions that are allowed by (single) gravitating solitons are just the trivial ones where $H=0$ and $F=0$. To demonstrate this point consider the following ansatz for the warp factors:

$$
a(w, \tau)=\frac{\alpha(\tau)}{\sqrt{\lambda^{2} w^{2}+1}}, \quad b(w, \tau)=\frac{\beta(\tau)}{\sqrt{\lambda^{2} w^{2}+1}}
$$

supplemented by the condition $\varphi=\varphi(w)$. Using Eq. (2.18) into Eqs. (2.6) and (2.7) it follows that $\mathcal{H}=0$ and $H^{\prime}=0$. But then the momentum constraint of Eq. (2.5) implies that, since $\dot{\varphi}=0$, also $F=0$. The requirement that the solution is separable demands, moreover, that all the pieces containing $H, F$, and their derivatives vanish in Eqs. (2.2), (2.3), (2.4), and (2.8). This condition translates, after some algebra, in the following pair of equations

$$
\dot{H}+H^{2}=0, \quad H F+H^{2}=0 .
$$

But since $F=0$ (because of the momentum constraint) it must also happen, according to Eq. (2.19) that $H=0$. Thus, $\alpha(\tau)$ and $\beta(\tau)$ can only be constants.

\section{TIME-DEPENDENT GRAVITATING MULTIDEFECTS}

The considerations developed in the previous section can be generalized to the situation where two scalar degrees of freedom are simultaneously present. The action to be discussed in the present section will therefore be

$$
\begin{aligned}
S= & \int d^{5} x \sqrt{|G|}\left[-\frac{R}{2 \kappa}+\frac{1}{2} G^{A B} \partial_{A} \varphi \partial_{B} \varphi\right. \\
& \left.+\frac{1}{2} G^{A B} \partial_{A} \chi \partial_{B} \chi-W(\varphi, \chi)\right] .
\end{aligned}
$$

Using the same conventions of the previous section the evolution equations only involving $\varphi, \chi$, and their first derivatives can be written, in the geometry defined by Eq. (1.5), as

$$
\begin{aligned}
& \dot{\varphi}^{2}+\dot{\chi}^{2}= \frac{1}{\kappa}\left[2 H(H+F)-2 \dot{H}-F^{2}-\dot{F}\right], \\
& \dot{\varphi} \varphi^{\prime}+\dot{\chi} \chi^{\prime}=\frac{1}{\kappa}\left[\dot{\mathcal{H}}-4 H^{\prime}+3 F \mathcal{H}\right], \\
& \varphi^{\prime 2}+\chi^{\prime 2}=\frac{3}{\kappa}\left(\mathcal{H} \mathcal{F}-\mathcal{H}^{\prime}\right) \\
&+\frac{b^{2}}{\kappa a^{2}}\left[(\dot{F}-\dot{H})+F^{2}+H F-2 H^{2}\right]
\end{aligned}
$$

The remaining three equations involve directly the scalar potential $W(\varphi, \chi)$ and they are

$$
\begin{gathered}
W(\varphi, \chi)=\frac{3}{2 \kappa a^{2}}\left(\dot{H}+2 H^{2}+H F\right) \\
-\frac{3}{2 \kappa b^{2}}\left(\mathcal{H}^{\prime}+4 \mathcal{H}^{2}-\mathcal{H} \mathcal{F}\right), \\
\ddot{\varphi}-\frac{a^{2}}{b^{2}} \varphi^{\prime \prime}+\left[(2 H+F) \dot{\varphi}-\frac{a^{2}}{b^{2}}(4 \mathcal{H}-\mathcal{F}) \varphi^{\prime}\right] \\
+a^{2} \frac{\partial W}{\partial \varphi}=0,
\end{gathered}
$$

$$
\begin{aligned}
\ddot{\chi}-\frac{a^{2}}{b^{2}} \chi^{\prime \prime}+\left[(2 H+F) \dot{\chi}-\frac{a^{2}}{b^{2}}(4 \mathcal{H}-\mathcal{F}) \chi^{\prime}\right] & \\
& +a^{2} \frac{\partial W}{\partial \chi}=0 .
\end{aligned}
$$

Consider, therefore, the following ansatz for the field profiles and for the warp factors:

$$
\begin{gathered}
\varphi(w, \tau)=\tilde{v} \sqrt{1+g(w, \tau)}, \\
\chi(w, \tau)=\tilde{v} \sqrt{1-g(w, \tau)}, \\
a(w, \tau)=\sqrt{1-g^{2}(w, \tau)}, \quad b(w, \tau)=\epsilon a(w, \tau),
\end{gathered}
$$

where $g(w, \tau)$ is a monotonic function which is continuous with its first and second derivatives with respect to $w$ and $\tau$. Furthermore, we do assume that $|g(w, \tau)|<1$ so that the square roots that appear ubiquitously in the ansatz are always well defined in the domain where $w$ and $\tau$ are allowed to vary. By inserting Eqs. (3.8) and (3.9) into Eqs. (3.2), (3.3), and (3.4) the following conditions on $g(w, \tau)$ can be explicitly obtained:

$$
\dot{g}^{\prime}=-\frac{3 g \dot{g} g^{\prime}}{1-g^{2}}, \quad g^{\prime \prime}=\dot{g}^{\prime} \frac{g^{\prime}}{\dot{g}}, \quad \ddot{g}=g^{\prime \prime} \frac{\dot{g}^{2}}{g^{\prime 2}} .
$$

To obtain Eq. (3.10) the arbitrary constant $v$ has been expressed in terms of $\kappa$ as $\kappa V^{2}=6$. The solution of Eq. (3.10) becomes then

$$
g(w, \tau)=\frac{\lambda(w+\tau)}{\sqrt{\lambda^{2}(w+\tau)^{2}+1}} .
$$

Equations (3.5), (3.6), and (3.7) can then be used to determine the scalar potential that becomes, within our conventions,

$$
\begin{aligned}
W(\varphi, \chi)= & W_{0}(\epsilon, \tilde{v})\left[\frac{6}{5} \tilde{v}^{2}\left(\varphi^{2}+\chi^{2}\right)-\left(\varphi^{4}+\chi^{4}\right)\right] \\
& +W_{1}(\epsilon, \tilde{v})\left(\varphi^{2}+\chi^{2}-2 V^{2}\right) \\
& \times\left[\frac{11}{8}\left(\varphi^{4}+\chi^{4}\right)-\tilde{v}^{2}\left(\varphi^{2}+\chi^{2}\right)\right]
\end{aligned}
$$

where 


$$
\begin{aligned}
& W_{0}(\epsilon, \tilde{v})=\frac{15 \lambda^{2}}{4 \kappa \tilde{v}^{4}}\left(\frac{1-\epsilon^{2}}{\epsilon^{2}}\right), \\
& W_{1}(\epsilon, \tilde{v})=\frac{\lambda^{2}}{2 \tilde{v}^{4}}\left(\frac{1-\epsilon^{2}}{\epsilon^{2}}\right) .
\end{aligned}
$$

It can be directly checked that Eqs. (3.12) and (3.13) solve directly Eqs. (3.5), (3.6), and (3.7). The physical situation described by the reported solution is the one where we have a kink and an antikink both time dependent. This aspect is illustrated by Fig. 5 where the functions $\varphi(w, \tau)$ and $\chi(w, \tau)$ are separately reported in the plot at the left and in the plot at the right.

As it was shown in the previous section, the one-field system only allows separable solutions which are trivial. If two fields are present the situation is different. Consider, for instance, the situation where the warp factors of the line element of Eq. (1.5) are parametrized as

$$
a(w, \tau)=\frac{\alpha(\tau)}{\sqrt{\lambda^{2} w^{2}+1}}, \quad b(w)=\frac{1}{\sqrt{\lambda^{2} w^{2}+1}},
$$

namely $b(w)$ is just static while the time dependence of $a(w, \tau)$ is separable. In this situation Eq. (3.3) is trivially satisfied while Eqs. (3.2) and (3.4) lead, respectively, to the following pair of conditions:

$$
\begin{gathered}
\varphi^{\prime 2}=\frac{3}{\kappa}\left(\mathcal{H}^{2}-\mathcal{H}^{\prime}\right)-\frac{1}{\kappa \alpha^{2}}\left[\dot{H}+2 H^{2}\right], \\
\dot{\chi}^{2}=\frac{2}{\kappa}\left(H^{2}-\dot{H}\right) .
\end{gathered}
$$

The potential can then be written as

$$
W(\varphi, \chi)=\frac{3}{2 \kappa a^{2}}\left(\dot{H}+2 H^{2}\right)-\frac{3}{2 \kappa b^{2}}\left(\mathcal{H}^{\prime}+3 \mathcal{H}^{2}\right) .
$$

There are now different possibilities and, for purposes of illustration, let us focus the attention on the simplest one,

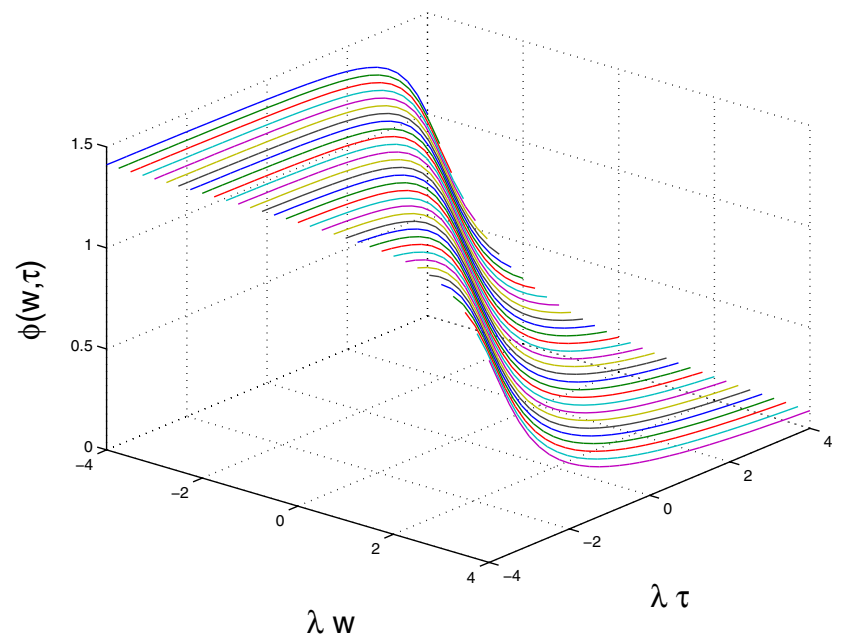

namely, the case where $\dot{H}+2 H^{2}=0$. In this situation the potential will be effectively independent on $\chi$ and the evolution of $\alpha(\tau)$ will be simply driven by the kinetic energy of $\chi$. In fact, Eqs. (3.15) and (3.16) reduce to

$$
\begin{gathered}
\varphi^{\prime}=\sqrt{\frac{3}{\kappa}} \frac{\lambda}{\lambda^{2} w^{2}+1}, \\
\dot{\chi}=\sqrt{\frac{6}{\kappa} H(\tau),}
\end{gathered}
$$

and $H(\tau)=(2 \tau)^{-1}$ which is, as expected, the expansion rate dictated typical of a Friedmann-Robertson-Walker (FRW) universe filled by a free (minimally coupled) scalar degree of freedom. Since $\dot{H}=-2 H^{2}$, Eq. (3.17) allows to determine also the potential of $\varphi$. The scalar profile $\varphi(w)$ and its related potential coincide, therefore, with the ones reported, respectively, in Eqs. (1.3) and (1.4).

\section{TRAVELING SOLITONS}

In this section we are going to discuss the case of traveling soliton solutions. More specifically it will be shown that, under certain conditions, every static solution cane be promoted, by Lorentz invariance, to a traveling soliton solution. To discuss this point it is useful to write some of the equations of the previous section in terms of the new boosted coordinates:

$$
u=\frac{w+U \tau}{\sqrt{1-U^{2}}}, \quad v=\frac{w-U \tau}{\sqrt{1-U^{2}}} .
$$

Consider therefore the line element of Eq. (1.5) with $a(w, \tau)=b(w, \tau)$. After few linear combinations it is easy to show, with simple algebra, that Eqs. (3.2), (3.3), and (3.4) translate in the following triplet of equations:

$$
\varphi_{u}^{2}+\chi_{u}^{2}=\frac{3}{\kappa}\left(h_{u}^{2}-h_{u u}\right)
$$

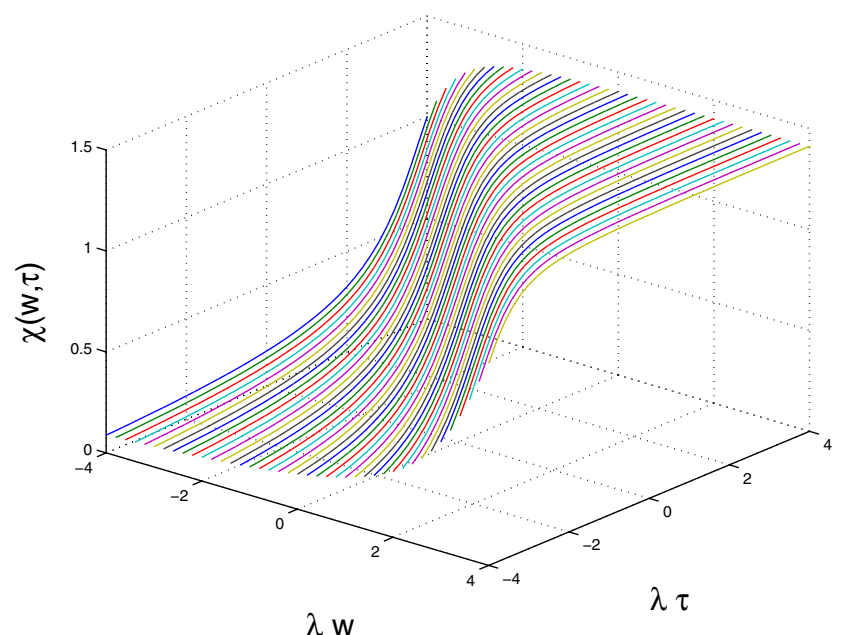

$\lambda w$

FIG. 5 (color online). The kink-antikink system of Eq. (3.8) in the case $\tilde{v}=1$ and always in natural gravitational units. 


$$
\begin{gathered}
\varphi_{v}^{2}+\chi_{v}^{2}=\frac{3}{\kappa}\left(h_{v}^{2}-h_{v v}\right), \\
\varphi_{u} \varphi_{v}+\chi_{u} \chi_{v}=\frac{3}{\kappa}\left(h_{u} h_{v}-h_{u v}\right),
\end{gathered}
$$

where the subscript denotes a partial derivation with respect to the corresponding variables; moreover $h=\ln a$. Equation (3.5) implies, in terms of the variables $o$ Eq. (4.1),

$$
\begin{aligned}
W(\varphi, \chi)= & -\frac{3}{2 \kappa}\left[\left(h_{u u}+h_{v v}+3 h_{u}^{2}+3 h_{v}^{2}\right)\right. \\
& \left.+2 \cosh 2 \alpha\left(h_{u v}+3 h_{u} h_{v}\right)\right],
\end{aligned}
$$

where $\cosh 2 \alpha=\left(1+U^{2}\right) /\left(1-U^{2}\right)$. It is finally easy to show that Eqs. (3.6) and (3.7) lead, respectively, to the following pair of equations:

$$
\begin{aligned}
\varphi_{u v}-\varphi_{u u} & -\varphi_{v v}-3\left(h_{u} \varphi_{u}+h_{v} \varphi_{v}\right) \\
& -3 \cosh 2 \alpha\left(h_{u} \varphi_{v}+h_{v} \varphi_{u}\right)+a^{2} \frac{\partial W}{\partial \varphi}=0, \\
\chi_{u v}-\chi_{u u}-\chi_{v v}-3\left(h_{u} \chi_{u}+h_{v} \chi_{v}\right) & \\
- & 3 \cosh 2 \alpha\left(h_{u} \chi_{v}+h_{v} \chi_{u}\right)+a^{2} \frac{\partial W}{\partial \chi}=0 .
\end{aligned}
$$

Suppose now that both $\varphi$ and $\chi$ are functions of $u$, i.e. $\varphi=$ $\varphi(u)$ and $\chi=\chi_{u}$. Then, Eqs. (4.3) and (4.4) will be satisfied provided $h=h(u)$, i.e. $a=a(u)$. Under these conditions the relevant set of equations to be solved becomes

$$
\begin{gathered}
\varphi_{u}^{2}+\chi_{u}^{2}=\frac{3}{\kappa}\left(h_{u}^{2}-h_{u u}\right), \\
W(\varphi, \chi)=-\frac{3}{2 \kappa}\left(h_{u u}+3 h_{u}^{2}\right) \\
\varphi_{u u}+3 h_{u} \varphi_{u}-a^{2} \frac{\partial W}{\partial \varphi}=0, \\
\chi_{u u}+3 h_{u} \chi_{u}-a^{2} \frac{\partial W}{\partial \chi}=0 .
\end{gathered}
$$

It can be easily seen that Eqs. (4.8), (4.9), (4.10), and (4.11) have the same form of the static solutions (in the two field case) when $a(w, \tau)=b(w, \tau)$. The derivative with respect to $w$ translates into a derivative with respect to $u$. So, every static solution can be boosted and the resulting metric and field profiles will still be a solution. The same conclusion can be reached if the boost is performed in the opposite direction. In this second case the field variables will only be functions of $v$ (and not of $u$ ) and the relevant equations can be obtained from Eqs. (4.8), (4.9), (4.10), and (4.11) by replacing $u \rightarrow v$ in the derivatives of $\phi, \chi$, and $h$.

As an example it is useful to consider the following solution of the above field equations in the case where both field profiles are only functions of, say, $u$ :

$$
\begin{aligned}
& \varphi(u)=\varphi_{0}\left\{[1+q(u)]^{3 / 2}+[1-q(u)]^{3 / 2}\right\}, \\
& \chi(u)=\chi_{0}\left\{[1+q(u)]^{3 / 2}-[1-q(u)]^{3 / 2}\right\},
\end{aligned}
$$

where $q(u)$ is a continuous and differentiable function of $u$; $\varphi_{0}$ and $\chi_{0}$ are both constants. As the function $g(w, \tau)$ introduced in the previous section, it will be required that $|q(u)|<1$ in the whole domain of definition. To satisfy the constraint of Eq. (4.4) it will be necessary that also $a=$ $a(u)$ and the static solution implies that the appropriate ansatz is nothing but

$$
a(u)=\frac{1}{\left(\lambda^{2 \mu} u^{2 \mu}+1\right)^{(1 / 2 \mu)}} .
$$

The function $q(u)$ is therefore determined to be

$$
q(u)=\frac{2}{\pi} \arctan \left[\lambda^{\mu} u^{\mu}\right], \quad \kappa \varphi_{0}^{2}=\frac{\pi^{2}}{12} \frac{2 \mu-1}{\mu^{2}}
$$

with $\chi_{0}=\varphi_{0}$. Equations (4.9), (4.10), and (4.11) allow to determine the potential:

$$
\begin{aligned}
W(\varphi, \chi)= & \frac{3 \lambda^{2}}{2 \kappa}\left[\sin ^{2} \sigma\right]^{(\mu-1) / \mu}\left[(2 \mu-1)-(2 \mu+3) \sin ^{2} \sigma\right] \\
& +\left[|\tilde{\varphi}+\tilde{\chi}|^{2 / 3}+|\tilde{\varphi}-\tilde{\chi}|^{2 / 3}-2\right] \mathcal{A}
\end{aligned}
$$

where, as usual, $\varphi=2 \varphi_{0} \tilde{\varphi}$ and $\chi=2 \varphi_{0} \tilde{\chi}$. The functions $\sigma(\tilde{\varphi}, \tilde{\chi})$ and $\mathcal{A}(\tilde{\varphi}, \tilde{\chi})$ appearing in Eq. (4.15) are defined, respectively, as

$$
\begin{aligned}
\mathcal{A}(\tilde{\varphi}, \tilde{\chi})= & \frac{18 b^{2} \varphi_{0}^{2} \mu}{\pi^{2}} \cos \sigma\left\{\sigma\left[\sin ^{2} \sigma\right]^{(\mu-2) / 2 \mu}[(\mu-1)\right. \\
& \left.\left.-(2 \mu+3) \sin ^{2} \sigma\right]+\frac{\mu}{2} \cos \sigma\left[\sin ^{2} \sigma\right]^{(\mu-1) / \mu}\right\}, \\
\sigma(\tilde{\varphi}, \tilde{\chi})= & \frac{\pi}{4}\left[|\tilde{\varphi}+\tilde{\chi}|^{2 / 3}-|\tilde{\varphi}-\tilde{\chi}|^{2 / 3}\right] .
\end{aligned}
$$

As expected this traveling solution has a static analog that has been discussed in [43].

To conclude the present section let us consider the possibility of having solutions that mix $u$ and $w$. There are, of course, trivial solutions to this problem, namely, the ones where the dependence of the various fields and of the warp factors depends either upon $(u+v)$ or upon $(u-v)$. In these two cases the resulting profiles are either completely static or completely time dependent.

The kind of mixed solutions which would be interesting to obtain would be the five-dimensional analog of the $(1+1)$-dimensional solutions of the sine-Gordon system that have been introduced in [1,2] and extensively discussed in [3]. Indeed, it is well known that there are no static two-kink solutions in the sine-Gordon system when only one scalar degree of freedom is present. However, two-kink solutions in which either kinks have 
arbitrary velocity (but at least one nonzero) can be constructed [1-3]. A typical example of this dynamics is the field profile given, in $(1+1)$ dimensions, by $4 \arctan [U \sinh (\gamma x) / \cosh (\gamma U t)]$ where $\gamma=\left(1-U^{2}\right)^{-1 / 2}$ and the two relevant coordinates have been denoted by $x$ (space) and $t$ (time). In this solution the kink approach the $x$ axis with velocity $U$, scatter elastically at $t=0$, and emerge from the interaction with a computable phase shift. Notice also that, for $t \rightarrow+\infty$ and $x \rightarrow+\infty$ the field profile becomes solely a function of $u=\gamma(x+U t)$. Conversely, for $x \rightarrow+\infty$ and $t \rightarrow-\infty$, the field profile becomes, asymptotically, only a function of $v=\gamma(x-U t)$.

In the five-dimensional warped geometries discussed in the present paper these solutions are rather hard to find and we cannot report any positive result. The main problem is represented by the structure of Eqs. (4.2), (4.3), and (4.4). Indeed it seems rather difficult to satisfy simultaneously the momentum constraint of Eq. (4.4) together with the conditions implied by Eqs. (4.2) and (4.3). In spite of this negative result it seems to be worthwhile to investigate further the possible solutions describing the elastic scattering of solitons in five dimensions. These solutions might have an intriguing cosmological interpretation.

\section{CONCLUDING REMARKS}

In the present paper time-dependent solitonic solutions have been discussed. It has been shown that these solutions exist both for the case of topological solitons and for the case of nontopological solitons. The discussion has been also generalized to include the possibility of multidefects, i.e. the situation where more than one defect is present in the system. In many respects the results of the present analysis are encouraging but still preliminary. They are encouraging since there exist solitonic profiles leading to time-dependent geometries that are diagonal and that have a well-defined $\mathrm{AdS}_{5}$ limit for large values of the bulk radius and for a fixed value of the conformal time coordinate. The reported results suggest several lines of possible developments. In the first place more realistic bouncing behaviors may be studied always in the case of five-dimensional warped geometries. Moreover, it is not excluded that different classes of solutions (like the ones related to elastic scattering of two profiles) may emerge.

The main aim of the present script has been to provide examples of thick defects that lead to solutions whose time-dependent evolution may be potentially relevant for cosmology. In this respect, an obvious extension of the reported considerations will be to study higherdimensional defects, like, for instance, Abelian vortices in six dimensions. These models are known to provide, at the static level, $\mathrm{AdS}_{6}$ geometries for large values of the bulk radius.

It would be finally interesting to compute the production of relic gravitons. Indeed, while at the static level the zero modes of the gravitons will certainly be localized owing to the $\mathrm{AdS}_{5}$ nature of the geometry, if the solutions exhibit time dependence the natural question to ask will be how many gravitons are produced by the time-dependent action of the gravitational field. This analysis requires necessarily a more realistic construction: it would not be enough, in the present approach, to patch different solutions since a more realistic implementation of the bouncing behavior is required. All these issues are beyond the aims of the present investigation.

\section{ACKNOWLEDGMENTS}

It is a pleasure to thank M. Halpern for valuable discussions.
[1] A. Seeger, H. Donth, and A. Kochendorfer, Z. Phys. 134, 173 (1953).

[2] J. K. Perring and T.H. Skyrme, Nucl. Phys. 31, 550 (1962).

[3] J. Rubinstein, J. Math. Phys. (N.Y.) 11, 258 (1970).

[4] R. Rajaraman, Solitons and Instantons (Elsevier, Amsterdam, 1982).

[5] S. Coleman, Aspects of Symmetry: Selected Erice Lectures (Cambridge University press, Cambridge, England, 1985).

[6] R. Rajaraman and E. Weinberg, Phys. Rev. D 11, 2950 (1975).

[7] C. Montonen, Nucl. Phys. B112, 349 (1976).

[8] T.D. Lee, Particle Physics and Introduction to Field Theory, Vol. 1 (Harwood, Academic, Chur, Switzerland, 1986), p. 117.

[9] A. Chodos, R. L. Jaffe, K. Johnson, C. B. Thorn, and V. F. Weisskopf, Phys. Rev. D 9, 3471 (1974).
[10] W.A. Bardeen, M.S. Chanowitz, S. D. Drell, M. Weinstein, and T.M. Yan, Phys. Rev. D 11, 1094 (1975).

[11] J. R. Morris, Phys. Rev. D 51, 697 (1995).

[12] D. Bazeia, L. Losano, and C. Wotzasek, Phys. Rev. D 66, 105025 (2002).

[13] A. de Souza Doutra, Phys. Lett. B 626, 249 (2005).

[14] M. Gremm, Phys. Lett. B 478, 434 (2000).

[15] M. Gremm, Phys. Rev. D 62, 044017 (2000).

[16] M. Giovannini, Phys. Rev. D 64, 064023 (2001).

[17] M. Giovannini, Phys. Rev. D 65, 064008 (2002).

[18] A. Kehagias and K. Tamvakis, Phys. Lett. B 504, 38 (2001).

[19] A. Kehagias and K. Tamvakis, Mod. Phys. Lett. A 17, 1767 (2002).

[20] M. Giovannini, Classical Quantum Gravity 20, 1063 (2003). 
[21] R. Koley and S. Kar, Classical Quantum Gravity 22, 753 (2005).

[22] S. Pal and S. Kar, Classical Quantum Gravity 23, 2571 (2006).

[23] M. Giovannini, Phys. Rev. D 75, 064023 (2007).

[24] K. Farakos and P. Pasipoularides, Phys. Lett. B 621, 224 (2005).

[25] C. Bogdanos, A. Dimitriadis, and K. Tamvakis, Phys. Rev. D 74, 045003 (2006).

[26] K. Farakos and P. Pasipoularides, Phys. Rev. D 75, 024018 (2007).

[27] K. Farakos, G. Koutsoumbas, and P. Pasipoularides, Phys. Rev. D 76, 064025 (2007).

[28] M. Giovannini, Phys. Rev. D 64, 124004 (2001).

[29] O. Corradini and Z. Kakushadze, Phys. Lett. B 494, 302 (2000).

[30] N. Deruelle and C. Germani, Nuovo Cimento Soc. Ital. Fis. B 118, 977 (2003).

[31] O. Corradini, Mod. Phys. Lett. A 20, 2775 (2005).

[32] N.E. Mavromatos and J. Rizos, Phys. Rev. D 62, 124004 (2000).

[33] M. Giovannini, Phys. Rev. D 63, 064011 (2001).
[34] N. E. Mavromatos and J. Rizos, Int. J. Mod. Phys. A 18, 57 (2003).

[35] M. Giovannini, H. Meyer, and M. E. Shaposhnikov, Nucl. Phys. B619, 615 (2001).

[36] O. Corradini, A. Iglesias, Z. Kakushadze, and P. Langfelder, Phys. Lett. B 521, 96 (2001).

[37] M. Giovannini, Phys. Rev. D 66, 044016 (2002).

[38] S. L. Parameswaran, S. Randjbar-Daemi, and A. Salvio, Nucl. Phys. B767, 54 (2007).

[39] S. Randjbar-Daemi, Mod. Phys. Lett. A 18, 2459 (2003).

[40] M. Giovannini and H. B. Meyer, Phys. Rev. D 64, 124025 (2001).

[41] S. Randjbar-Daemi and M. Shaposhnikov, Nucl. Phys. B645, 188 (2002).

[42] M. Giovannini, Phys. Rev. D 63, 085005 (2001).

[43] M. Giovannini, Classical Quantum Gravity 23, L73 (2006).

[44] L. Parker, Nature (London) 261, 20 (1976).

[45] L. Parker, Phys. Rev. 183, 1057 (1969).

[46] M. Gasperini, M. Giovannini, and G. Veneziano, Phys. Lett. B 569, 113 (2003). 